\title{
Monitoring the Nervous System for Anesthesiologists and Other Health Care Professionals, Second Edition
}

\author{
Antoun Koht, Tod B. Sloan, J. Richard Toleikis (Editors). Springer, 2017, 722 pages. \\ ISBN 978-3-319-46540-1
}

\author{
Oana Predescu, MD, MSc, FRCPS
}

Received: 12 March 2018/Revised: 19 March 2018/Accepted: 21 March 2018/Published online: 2 April 2018

(c) Canadian Anesthesiologists' Society 2018

The presence of a neuro-trained anesthesiologist and intensivist offers potential benefit in optimizing patient safety and reducing neuronal insults - not only in the neurosurgical operating room and intensive care unit but in orthopedic, ear/nose/throat, and cardiovascular surgical settings. Furthermore, with plans moving forward on establishing an accredited training program in perioperative neuroscience as part of a neuroanesthesiology fellowship program, ${ }^{1}$ the second edition of the Monitoring the Nervous System for Anesthesiologists and Other Health Care Professionals comes close to being considered a mandatory basic textbook.

This comprehensive, 700-page tome leads the reader through each current intraoperative neuro-monitoring modality in an easy-to-read format and well-structured chapters. Professionals from numerous specialties anesthesiologists, orthopedic surgeons, neurosurgeons, neurophysiologists, neurologists - collaborate in convincing the student that not only well-known applied technology, but also teamwork, are what improve patient care and ensure good outcomes.

The present edition appears five years after its first edition, with updated references and some new chapters. Similar to the first edition, the 2017 textbook is structured in four sections.

The first section "Monitoring Techniques," describes each modality in length, with essential clinical details, including indications for the utilization of each measurement, contraindications, and possible complications. With rapid progress in the performance of

O. Predescu, MD, MSc, FRCPS (ه)

McGill University, Montreal, QC, Canada

e-mail: oanadp@mac.com complex technologies, I would have liked to have seen references regarding the reliability and validity of each measuring instrument, as well as data from the literature about the specificity and sensitivity of each measurement. A novel chapter regarding electrical interference with intraoperative neuro-monitoring instruments explains, with illustrative videos, the usually forgotten physics of electrical interactions that occur routinely in the operating room.

The second section, "Anesthetic Considerations," includes a chapter that synthesizes the information on anesthetic management during intraoperative electrophysiological monitoring, including influences of each anesthetic drug on the monitor's readings. Although the content of this chapter slightly overlaps descriptions in Sections 1 and 3, it, in fact, is useful for highlighting the principles of neurological monitoring and helps the reader retain information. The added videos of performing regional anesthesia techniques for awake neurosurgical procedures are valuable adjuncts to the text.

The third section, "Clinical Applications," the largest section of the textbook, starts with a basic algorithm for detecting the cause of deterioration observed during intraoperative neuro-monitoring. The algorithm synthesizes knowledge acquired from previous sections of the book. With its "learning key points" at the beginning of each chapter and its well-chosen, relevant clinical cases, explanatory videos, and review questions at the end of each subject, the Clinical Applications section deepens the understanding of anesthetic considerations. It accomplishes it by reinforcing the importance not only of the scientific, theoretical aspects of intraoperative monitoring but also the data acquisition and interpretation challenges in each particular context. It 
also acknowledges the significance of collaboration and communication among team members during all types of surgery that present a risk of injury to the nervous system.

The final section, "Intensive Care," is a brief description of three neuro-trauma intensive care unit (ICU) patients with their treatment and monitoring. References to various other chapters in Sections 1 and 2 complete the clinical picture and our understanding of the subject. It is a short chapter, not exhaustively treated, which is understandable as the focus of this book is on intraoperative monitoring, not neuro-ICU monitoring.

The book is well referenced, with mostly up-to-date literature and guidelines, although the 2016-updated Brain Trauma Foundation Guidelines ${ }^{2}$ are missing from the bibliography. Nevertheless, the text is complemented by remarkably high-quality pictures and explicative videos.

In the neuro-anesthesia literature, most textbooks focus on either neuro-monitors or intraoperative neuromonitoring techniques. This unique, high-value textbook emphasizes the importance of multimodal neuromonitoring adapted to each surgical procedure, offering both a description of the instrument used and its direct clinical application. This approach promotes better understanding of the operating room reality and the means available to improve patients' safety and clinical outcomes.

Monitoring the Nervous System for Anesthesiologists and Other Health Care Professionals will be an important and helpful learning reference for anesthesia trainees as well as others training in surgical specialties that require neuro-monitoring.

Conflicts of interest None declared.

Editorial responsibility This submission was handled by Dr. Hilary P. Grocott, Editor-in-Chief, Canadian Journal of Anesthesia.

\section{References}

1. Society for Neuroscience in Anesthesiology and Critical Care. International Council on Perioperative Neuroscience Training (ICPNT). Charter. Amended January 21, 2018. Available from URL: http://www.snacc.org (accessed March 2018).

2. Guidelines for the Management of Severe Traumatic Brain Injury, Fourth Edition. Available from URL: www.braintrauma.org/coma/ guidelines (accessed March 2018). 